\title{
Neuroprotective Effects of Tea against Cadmium Toxicity
}

\author{
${ }^{1}$ Areba G.O., ${ }^{2}$ Khalid R., ${ }^{3}$ Ngure R.M., ${ }^{4}$ Maloba F., ${ }^{5}$ Nyaga N., ${ }^{6}$ Moseti K.O., ${ }^{7}$ Ngotho M., \\ ${ }^{6}$ Wanyoko J.K., ${ }^{8}$ Karori S.M. and ${ }^{9 *}$ Wachira F.N.
}

${ }^{1}$ Department of Veterinary Pathology, Microbiology and Parasitology, Egerton University, Kenya; ${ }^{2}$ Department of Ophthalmology, University of Cologne, Germany; ${ }^{3}$.Department of Veterinary Clinical Studies, Egerton University, Kenya; ${ }^{4}$ Department of Zoological Sciences, Kenyatta University, Kenya. ${ }^{5}$ Department of Veterinary Anatomy and Physiology, Egerton University, Kenya; ${ }^{6}$ Tea Research Institute of Kenya, Kenya; ${ }^{7}$ Mount Kenya University, Kenya; ${ }^{8}$ Department of Biochemistry and Molecular Biology, Egerton University, Kenya,; ${ }^{9}$ Department of Life Sciences, South Eastern Kenya University, Kenya.

*Corresponding Author: Wachira F.N., Department of Life Sciences, South Eastern Kenya University, Kenya.

Submission Date: August 5, 2019. Acceptance Date: December 24 ${ }^{\text {th }}$, 2019. Publication Date: December $30^{\text {th }}, 2019$

Citation: Areba GO, Khalid R, Ngure RM, Maloba F, Nyaga N, Moseti KO, Ngotho M, Wanyoko JK, Karori SM, Wachira FN. Neuroprotective Effects of Tea against Cadmium Toxicity. Bioactive Compounds in Health and Disease 2019; 2(12): 230-246. DOI: https://doi.org/10.31989/bchd.v2i12.684

\begin{abstract}
Background: Cadmium $(\mathrm{Cd})$ is a common pollutant and potential neuro-toxicant to humans. The main treatment for heavy metal toxicity is chelation therapy which is however replete with grave side effects. This study was designed to determine the neuroprotective effects of extracts of the tea beverage on experimentally induced cadmium toxicity in the brain of rats. Cadmium as $\mathrm{CdCl}_{2}$ was administered subcutaneously while tea was given orally.
\end{abstract}

Methods: Healthy Wister rats were used to study the effects of co-administration of $\mathrm{Cd}$ and tea extracts on the brain. Cadmium was injected subcutaneously while tea was administered orally to the rats. Brain tissue from euthanized rats was assayed for Zinc Fingers and Homeoboxes Protein 1 (ZHX1), reduced glutathione (GSH), and lipid peroxidation markers Thiobarbituric Acid Reactive Substances (TBARS). Neurohistochemical and histopathological studies were also carried out on the brain tissues of the rats.

Results: Cadmium significantly induced neuronal damage exhibited by a significant $(p<0.05)$ decrease in ZHX1 in the brain tissue, significant $(p<0.05)$ increase in TBARS, as well as significant $(p<0.05)$ increase in GSH implying an impaired antioxidant defense system. Co- 
administration of $\mathrm{Cd}$ with black or green tea extracts resulted in a significant decrease in lipid peroxidation as well as maintenance of GSH and ZHX1. The neurohistochemical and histopathological studies in the brain of the rats indicated that the tea extracts significantly reduced $\mathrm{CdCl}_{2}$ toxicity and preserved the normal histological architecture of the brain tissues.

Conclusion: This paper reports for the first time the efficacy of tea extracts in protecting rats from cadmium induced toxicity and disturbances of antioxidant defense system in the brain.

Key words: Tea; flavonoids; Cadmium; neurotoxicity; Chelating agents.

\section{INTRODUCTION}

Heavy metal toxicity is one of the oldest environmental problems and remains a serious health concern today. The general public is exposed to Cadmium $(\mathrm{Cd})$ which is a common heavy metal toxicant in the environment through ambient air, drinking water, food, cigarette smoking, industrial materials, consumer products, burning of fossil fuels and waste materials as well as use of high phosphate and sewage sludge fertilizers [1]. Heavy metals cause oxidative deterioration of biological macromolecules by binding to DNA and nuclear proteins [2]. Cadmium is a neurotoxicant that has been shown to affect developing cortical cells on immature hippocampal cells and on developing brain [3,4]. Although entry of Cd into the adult central nervous system (CNS) is limited, developmental neurotoxicity may occur as a result of blood brain barrier (BBB) immaturity [3, 4]. Increased levels of $\mathrm{Cd}$ have however been shown to impair the functionality of BBB [5]. Many effects of Cd result from interactions with necessary micro- and macro-elements, especially $\mathrm{Ca}, \mathrm{Zn}, \mathrm{Cu}$, iron, and $\mathrm{Se}[6]$.

Extensive studies have reported that exposure to high $\mathrm{Cd}$ levels typically results in the excessive production of reactive oxygen species (ROS) by up-regulation of the expression of NADPH oxidase II and the depletion of the antioxidant molecule, glutathione (GSH) [7]. In turn this results in elevated oxidation of lipids, proteins and nucleic acids in various tissues such as lung, brain, kidney, liver, erythrocyte and testes [8].

Zinc Fingers and Homeoboxes Protein 1 (ZHX1) regulates transcription by playing like a bridging molecule between DNA methyltransferase B (DNMT3B) and other co-repressor proteins that form a universal repressor complex that enhances transcription repression [9]. Cd has been reported to induce inhibition of zinc finger proteins through iso-structural substitution, and replacement of zinc with altered geometry [10]. There is also increasing evidence that $\mathrm{Cd}$ binds to zinc finger proteins reducing their affinity to DNA binding sites and impairing DNA damage recognition in the case of DNA repair proteins [11]. Consequently, there is now growing evidence that $\mathrm{Cd}$ toxicity is an etiological factor in various neurodegenerative disorders [12]. Cadmium produces neuropathological and neurochemical alterations in the CNS that lead to irritability and hyperactivity [13]. In addition, Cd-induced neurotoxicity has been associated with astrogliosis with the concomitant secretion of neurotoxic factors and an enhanced expression of the glial fibrillary acidic protein (GFAP). This is thought to interfere with the necessary signaling between astrocytes and neurons or synapses further aggravating the neurodegenerative process $[14,15]$.

Although there is no available antidote that could instantaneously remove $\mathrm{Cd}$ from the blood and soft tissues [16], its poisoning has classically been treated by synthetic chelating agents such 
as calcium disodium ethylenediamine tetra acetic acid ( $\left.\mathrm{CaNa}_{2} \mathrm{EDTA}\right)$, British Anti Lewisite (BAL), sodium 2,3-dimercaptopropane 1-sulfonate (DMPS) and meso 2,3-dimercaptosuccinic acid (DMSA) among others [17, 2]. These synthetic chelating agents have however been associated with grave side effects such as the binding of essential metals within the system which significantly reduces their efficacy, and mobilization of heavy metals towards the central nervous system which aggravates the situation further. Besides, these agents have to be intravenously administered by a medical practitioner which makes it difficult for the patient to resort to self aid and they are contraindicated for various cases of heavy metal toxicity [18, 17]. It seems then that these synthetic chelating agents have several unacceptable side effects and drawbacks that make them unsafe and ineffective treatment and management strategies for $\mathrm{Cd}$ poisoning in modern medicine. There is therefore a need for an alternative, effective and safe treatment and management strategy for Cd toxicity.

The therapeutic use of antioxidants from diet appears to be gaining a lot of popularity due to their ability to ameliorate a range of conditions including neurodegenerative diseases with very low incidence of toxicity even at exceptionally high dosages [19]. Natural chelating-antioxidants may therefore offer a novel therapeutic strategy for the management of $\mathrm{Cd}$ poisoning. Tea (Camellia sinensis) has particularly been shown to be pharmacologically active and to have a host of health benefits on humans including anti-inflammatory and anti-microbial properties, scavenging of free radicals and prevention of diet induced obesity by modulation of lipid metabolism [20,21]. The potential health benefits of this beverage have been ascribed to its high levels of polyphenols, including flavonoids such as catechins, thearubigins and theaflavins [22, 23]. Flavonoids preferentially enter the hydrophobic core of the cell membrane where they exert a membrane-stabilizing effect by modifying the lipid packing order [24, 25]. Numerous studies have documented the efficacy of polyphenols in the management of neurodegenerative diseases $[26,27]$. However despite such potential, there is still a paucity of data that compares the ameliorative properties of black and green tea with synthetic chelating agents on $\mathrm{Cd}$ induced toxicity in suitable animal models. This study therefore aimed at comparing the neuroprotective effects of tea and EDTA on cadmium induced neurotoxicity. We hypothesized that tea polyphenols would be better chelating-antioxidants than the conventional synthetic chelating agents due to their structural features which include the aromatic ring structure coupled with the hydroxyl groups in the rings and conjugated double bond system.

\section{MATERIALS AND METHODS}

\section{Materials}

Analytical grade Cadmium chloride, Na2 EDTA, 2-thiobarbituric acid and 5'5'-dithiobis-(2nitrobenzoic-acid) were used in this study (Sigma, St. Louis MO, USA).

\section{Animals}

Healthy 6 months male drug/test naïve Wister rats all weighing between 300-400 g were used in this study. The rats were reared at the Institute for Primate Research (IPR), Kenya. All experimental procedures and protocols involving experimental animals were reviewed and approved for adherence to Standard Operating Procedures (SOP) of the Institutional Animal Care and Use Committee (IACUC) of the Institute of Primate Research (IPR), with approval number IRC/08/13. 


\section{Tea Samples}

Fresh tea leaves comprised of the youngest two leaves and a bud were harvested from a plot of a pure stand of cultivar TRFK 6/8 and used in this study. Tea processed from this cultivar has been shown to be high in quality when compared to teas from other cultivars and to have the highest levels of total polyphenols, theaflavins, and antioxidant activity among other properties [28]. The cultivar was grown at the Tea Research Institute (TRI), Timbilil Estate, Kericho, Kenya (latitude $0^{\circ} 22$ 'S, longitude $35^{\circ} 21^{\prime} \mathrm{E}$, altitude $2180 \mathrm{~m}$ a.m.s.l). Black and green tea samples were processed from the harvested leaf at the TRI miniature factory using standard TRI optimized manufacturing procedures.

\section{Experimental design}

A total of twenty five (25) experimental rats were housed in standard mice cages in a controlled environment and provided ad libitum pellet food and water during which each rat was treated once using $0.1 \mathrm{~mL}$ of $1 \%$ Ivermectin (Ivomec $®$ ) equivalent to $1 \mathrm{mg}$ per rat during the first week in order to exclude any helminthes infestation. After acclimatization for two weeks, the twenty five (25) rats were randomly divided and caged into five (5) groups of five (5) animals each and treated as follows;

Group I: Control rats which received daily subcutaneous injection of isotonic saline for six weeks

Group II: This group received subcutaneous injections of $\mathrm{Cd}$ as $\mathrm{CdCl}_{2}(2 \mathrm{mg} / \mathrm{kg}$ bw/day) in isotonic saline daily for 6 weeks

Group III: This group received a subcutaneous injection of $\mathrm{CdCl}_{2}(2 \mathrm{mg} / \mathrm{kg}$ bw/day) daily followed by an oral intra-gavage administration of aqueous black tea extracts- BTE (400 mg/kg bw/day) for six weeks

Group IV: This group received a subcutaneous injection of $\mathrm{CdCl}_{2}(2 \mathrm{mg} / \mathrm{kg}$ bw/day) daily followed by an oral intra-gavage administration of aqueous green tea extracts- GTE (400 mg/kg bw/day) for six weeks

Group V: This group received a subcutaneous injection of $\mathrm{CdCl}_{2}(2 \mathrm{mg} / \mathrm{kg}$ bw/day) daily followed by a subcutaneous injection of aqueous $\mathrm{Na}_{2}$ EDTA $(200 \mathrm{mg} / \mathrm{kg}$ bw/day) for six weeks

\section{Tissue Preparation for Analysis}

At the end of the experimental period, the animals in different groups were euthanized using $\mathrm{CO}_{2}$. Blood was collected and centrifuged for the separation of serum. The brain was excised and divided into two halves. One half was homogenized on ice cubes $\left(4^{\circ} \mathrm{C}\right)$ in a solution containing $0.5 \mathrm{mls}$ of $0.25 \mathrm{M}$ sucrose, $5 \mathrm{mM}$ Hepes-Tris $\mathrm{pH} 7.4$ with protease inhibitor cocktail to a final concentration of $10 \%(\mathrm{w} / \mathrm{v})$. The homogenates were centrifuged then aliquoted in triplicates into $1.5 \mathrm{ml}$ cryovials to avoid repeated freeze-thaw processes and stored at $-80{ }^{\circ} \mathrm{C}$ until analysis. The other half of the brain tissue was used for histological and immunohistochemical studies.

\section{Determination of Glutathione}

Glutathione assay was performed using the method of Rahman et al., [29] with modifications as described by Rashid et al., [30]. 


\section{Zinc Fingers and Homeoboxes Protein 1 (ZHX1)}

Quantitative determination of rat zinc fingers homeoboxes protein 1 (ZHX1) was performed using a commercially available ELISA kit $\left(\mathrm{CUSABIO}^{\odot}\right.$, Biotech Limited, China) according to the manufacturer's instructions.

\section{Thiobarburic Acid Reactive Substances (TBARS) Assay}

The TBARS assay was performed using a commercially available Kit (QuantiChrom ${ }^{\mathrm{TM}}$, Gentaur Molecular Products, Kampenhout, Belgium) according to the manufacturer's instructions. This assay is based on the reaction of malondialdehyde (MDA), a principle TBARS with Thiobarburic acid (TBA) to form a pink chromogen attributable to an MDA-TBA 2 adduct. The colour intensity at $535 \mathrm{~nm}$ is directly proportional to TBARS concentration in the sample.

\section{Hematoxylin and Eosin (H\&E) Staining}

Following rat sacrifice, brains were excised and one half fixed in $10 \%$ formal saline, embedded in paraffin wax and the paraffin blocks sectioned at a thickness of 3 to $4 \mu \mathrm{m}$. Subsequently, the sections were stained with Hematoxylin and Eosin (H\&E) and examined histologically under the light microscope as described by Rashid et al., [30].

\section{Immunohistochemistry}

Immunoperoxidase staining of $5 \mu \mathrm{m}$ fixed cryostat sections of the cerebral cortex and the cerebellum were carried out with GFAP antibody as described previously [31,32,33]. The sections were histologically examined under the light microscope.

\section{Data Analysis}

Statistical analyses were performed using Graph pad Prism software version 5. Results were given as mean \pm standard error of mean $(\mathrm{SEM})$ with significance level set at $\mathrm{p}<0.05$. One way analysis of variance (ANOVA) was used to test for differences between the means of GSH, ZHX1 and TBARS and post hoc tests done to evaluate the differences among the group means.

\section{RESULTS}

\section{Animal health}

No adverse events on the physical health of the test animals were reported during the study.

\section{Glutathione}

To elucidate the mechanism by which $\mathrm{CdCl}_{2}$ induced cytotoxicity, we investigated its effects on GSH, one of the major intracellular thiol antioxidants. There was a significant $(P<0.05)$ increase in the cellular GSH levels in homogenized rat brain tissues after exposure to $\mathrm{CdCl}_{2}$ (Figure 1). Interestingly, tea and EDTA significantly $(P<0.05)$ reduced $\mathrm{CdCl}_{2}$ induced GSH up-regulation in rats. However, both tea extracts were found to give better results than EDTA. 


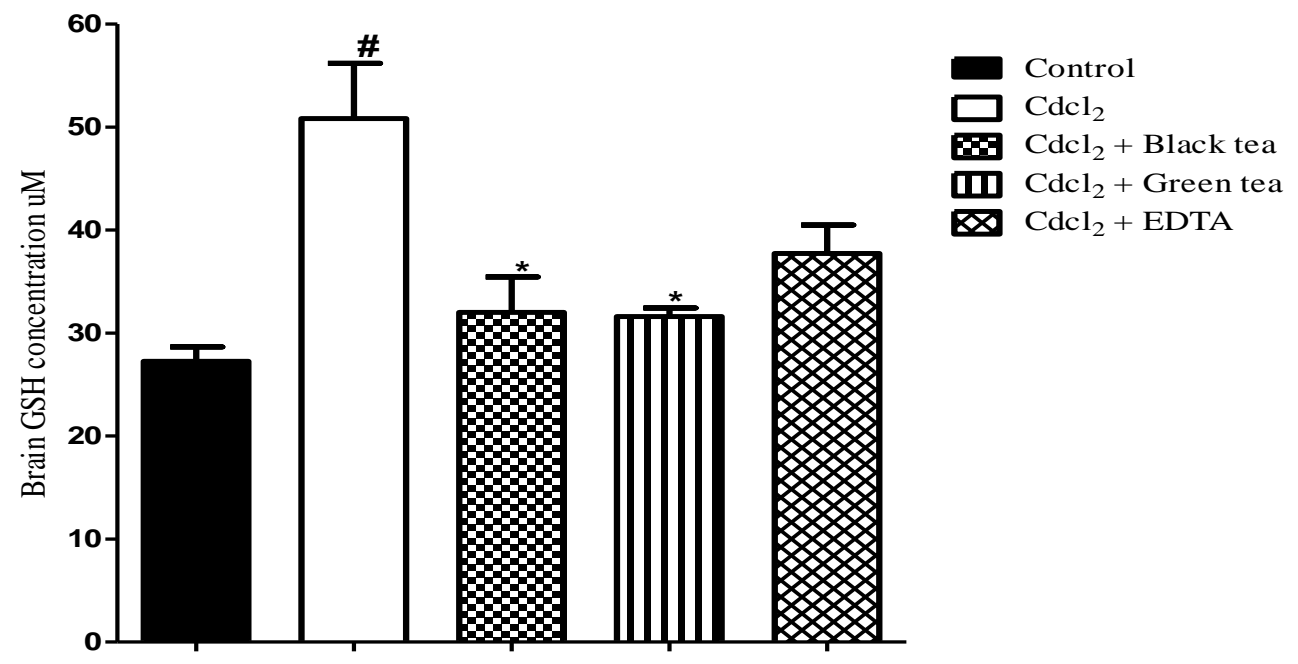

Figure 1: Effect of $\mathrm{CdCl}_{2}$ exposure with or without chelating antioxidants intervention on brain GSH levels in rats.

\# Statistically significant versus controls.

* Statistically significant versus $\mathrm{CdCl}_{2}$ group.

\section{Zinc fingers and homeoboxes I (ZHXI)}

In the present study, $\mathrm{CdCl}_{2}$ exposure to rats induced significant $(P<0.05)$ reduction in brain ZHX1 levels. Both tea extracts and EDTA effectively restored $(P<0.05)$ brain ZHX1 levels against $\mathrm{CdCl}_{2}$ induced neurotoxicity (Figure 2). Remarkably, performance of green tea in alleviating severe $\mathrm{CdCl}_{2}$ induced down regulation of $\mathrm{ZHX} 1$ in the brain was comparable with that of EDTA with no significant differences $(P>0.05)$ observed between the two groups.

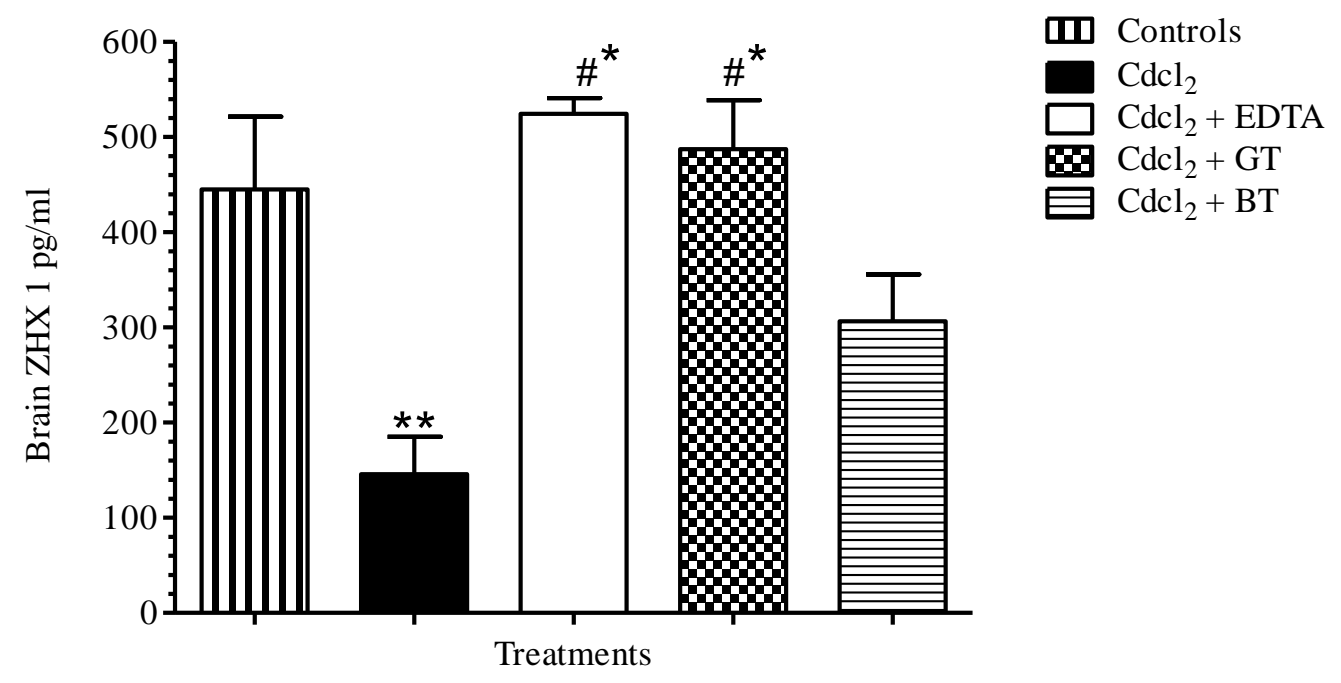

Figure 2: Effect of $\mathrm{CdCl}_{2}$ exposure with or without chelating antioxidants intervention on total brain ZHX1 levels in rats.

** denotes a statistically significant reduction of ZHX1 when compared to the control rats.

\#* denotes a statistically significant alleviation of the severe $\mathrm{CdCl}_{2}$ induced down regulation of $\mathrm{ZHX} 1$ in the brain when compared to $\mathrm{CdCl}_{2}$ challenged rats. 


\section{Thiobarburic acid assays (TBARS)}

Brain tissue levels of TBARS, measured as lipid peroxidation end product malondialdehyde (MDA) were significantly $(P<0.05)$ up-regulated in the $\mathrm{CdCl}_{2}$ treated group when compared with the control rats (Figure 3). Black and green tea significantly $(P<0.05)$ inhibited $\mathrm{CdCl}_{2}$ induced MDA up regulation in rats while the conventional chelating agent EDTA failed to exhibit any protective effect against accumulation of lipid peroxidation products.

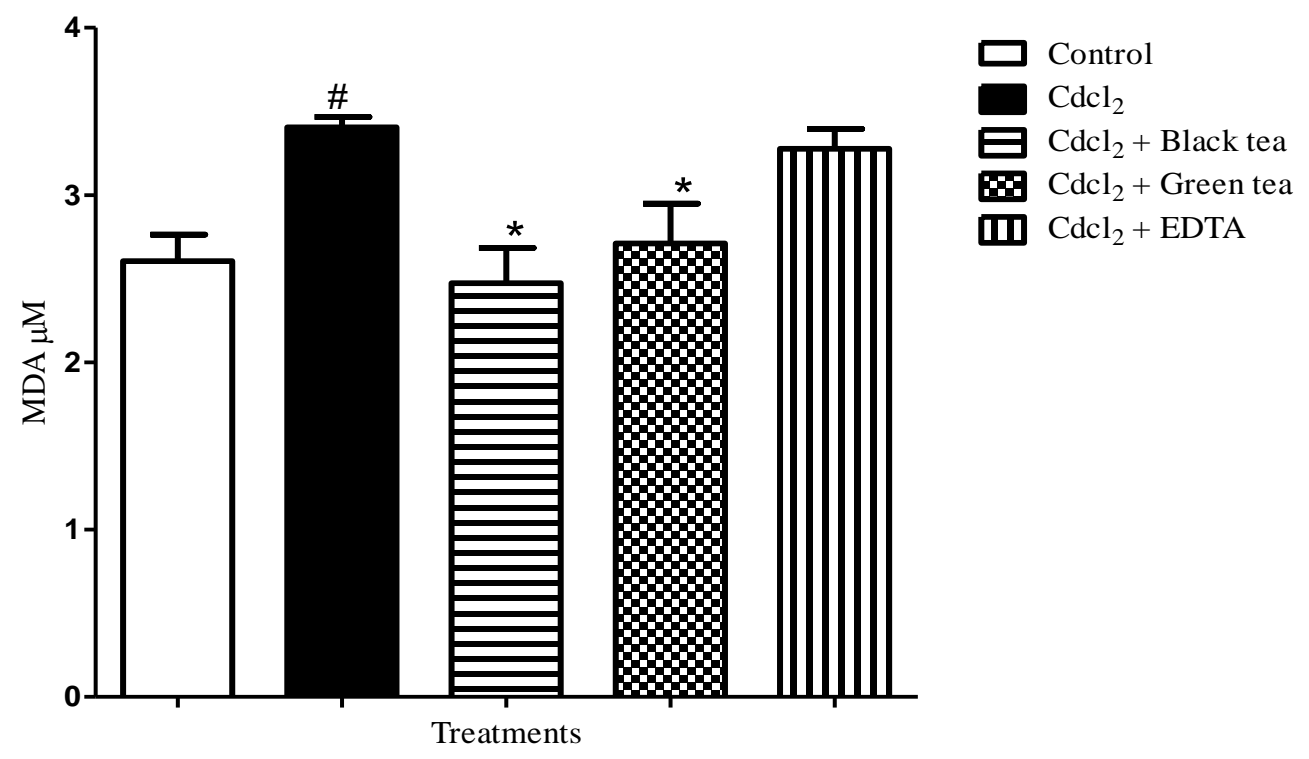

Figure 3: Effect of $\mathrm{CdCl}_{2}$ exposure with or without chelating antioxidants intervention on brain MDA levels in rats.

* indicates that both black and green tea are statistically significantly $(\mathrm{P}<0.05)$ different when compared to $\mathrm{CdCl}_{2}$ challenged rats.

\# denotes that MDA up-regulation is statistically significant $(\mathrm{P}<0.05)$ when compared to Control rats.

\section{Histopathology}

A representative photomicrograph showing histopathological changes in the brains of the control, $\mathrm{Cd}$ and tea treated rats is presented in Figure 4. Brains of $\mathrm{CdCl}_{2}$ treated rats were characterized by a marked presence of lymphocytic inflammatory changes and brain necrosis (Plate B). Brain tissues of rats treated with both $\mathrm{CdCl}_{2}$ and $\mathrm{Green}$ Tea (Plate C), $\mathrm{CdCl}_{2}$ and Black Tea (Plate D) showed a marked reduction of inflammatory cells and a near normal architecture of the brain tissue. 


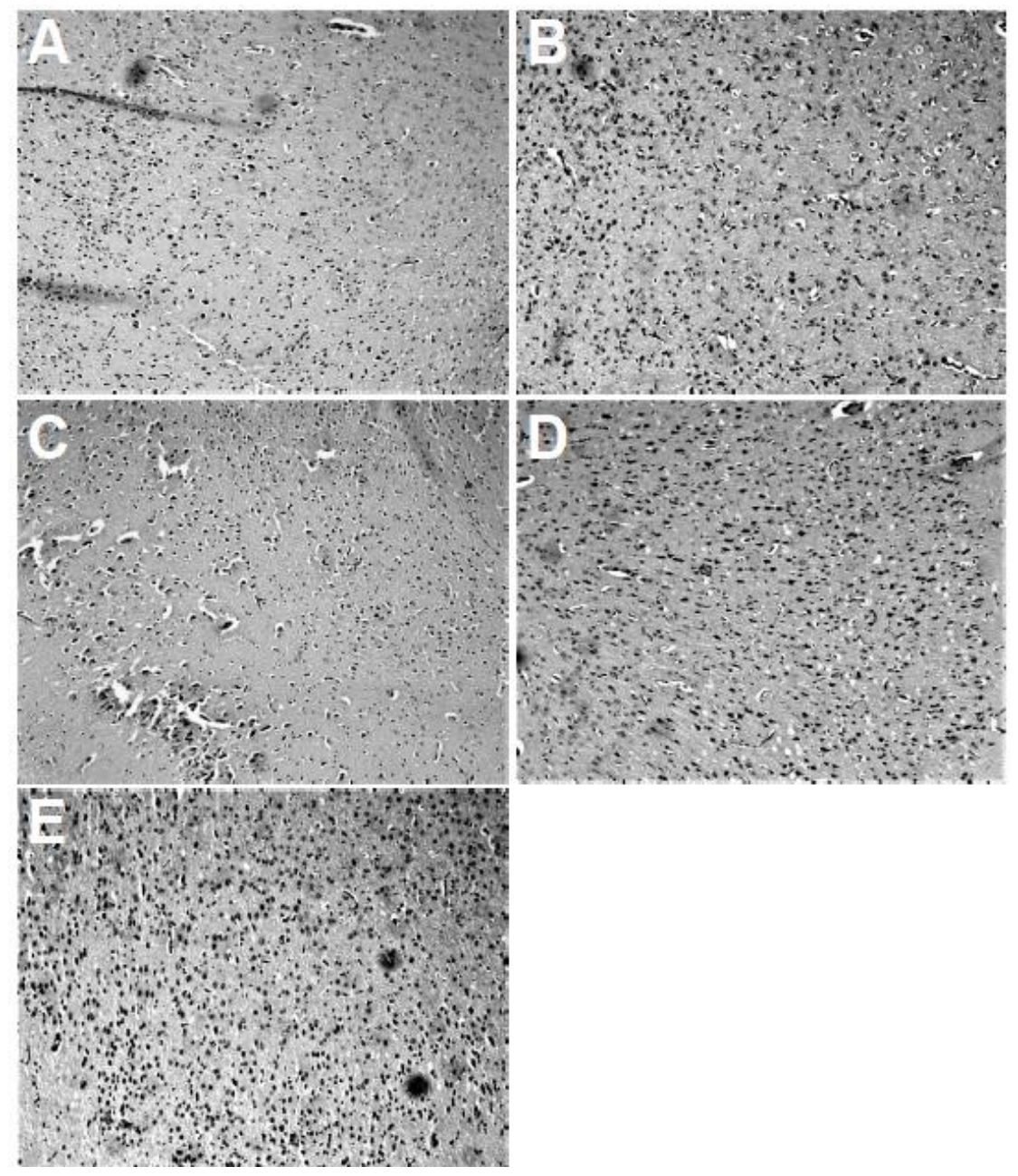

Figure 4: Representative photomicrograph showing histopathological changes in the rat brain (H\&E, 100X A and B; 200X C and D) of control and experimental rats. Plate A: Brain of normal healthy rat (control), Plate B: Brain of $\mathrm{CdCl}_{2}$ treated rat, Plate $\mathrm{C}$ : Brain tissue of rat treated with $\mathrm{CdCl}_{2}$ and GTE, Plate D: Brain tissue of rat treated with $\mathrm{CdCl}_{2}$ and BTE, Plate E: Brain tissue of rat treated with $\mathrm{CdCl}_{2}$ and EDTA

\section{Immunohistochemistry}

Representative photomicrographs showing immunohistochemical changes in the brains of the control, $\mathrm{Cd}$ and tea treated rats are presented in Figure 5. Healthy rats exhibited normal brain immunohistochemistry (Plate A). $\mathrm{CdCl}_{2}$ challenged brain sections exhibited increased GFAP staining characterized by round shaped cell bodies and relatively small number of fibrous processes. Additionally, there was marked increase in the intensity of immunostaining and astrocytic proliferation (Plate B). The brains of Cadmium and Black tea treated rats showed a mild reduction of GFAP staining with a relative increase in the fibrous processes (Plate $\mathrm{C}$ ). Cadmium and green tea treated rats had brains with significant reduction of GFAP staining and increased fibrous processes (Plate D). The Cadmium and EDTA treated rats similarly had brains with significantly reduced GFAP staining and with increased fibrous processes (Plate E). Besides the reduction in GFAP staining, treatment of the rats with tea caused relaxation of the astrocytes (Plates $\mathrm{C}$ and D) 

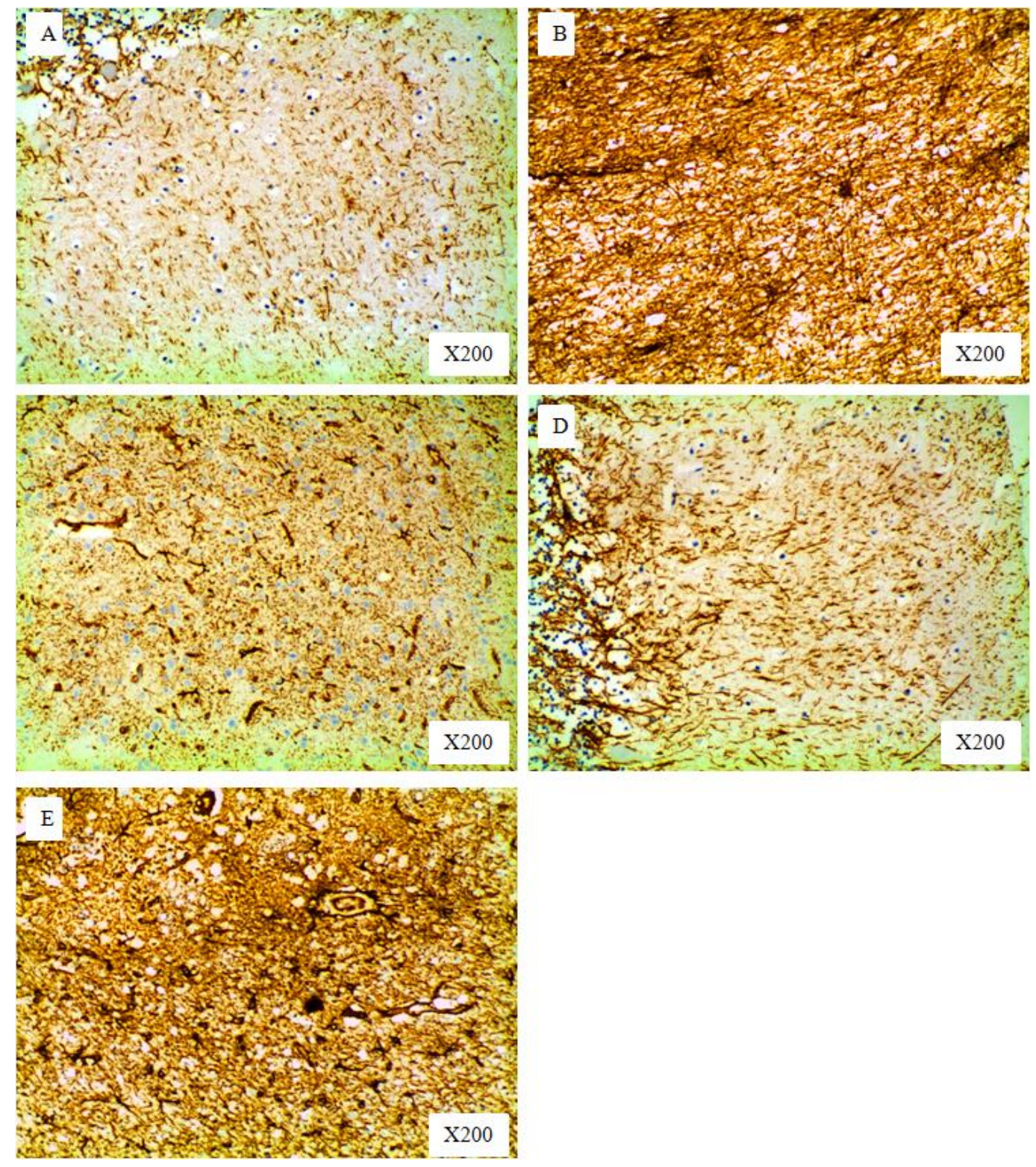

Figure 5: Representative photomicrographs showing the effects of Cd, BTE, GTE and EDTA on the immunohistochemistry of the brain. Plate A: Normal brain of healthy rat (control), Plate B: $\mathrm{CdCl}_{2}$ challenged brain, Plate C: Brain section of Cadmium and Green tea treated rat, Plate D: Brain section of Cadmium and Black tea treated rat, Plate E: Brain section of Cadmium and EDTA treated rat.

\section{DISCUSSION}

Cadmium (Cd) induced toxicity is a notorious occurrence, whose effective treatments are still limited. Given that the risk of human exposure to $\mathrm{Cd}$ is persistently growing due to its prevalence in the environment and the absence of decomposable processes [34], there is a strong impetus to develop effective therapeutic strategies for managing Cd toxicity. While there is no consensus on the efficiency of chelating therapy for $\mathrm{Cd}$ induced toxicity, some scholars hold the view that $\mathrm{Cd}$ chelation may well intensify its elimination, thus reducing its toxic effects $[35,36]$. Interestingly, the co-administration of chelating agents and antioxidants has been associated with better effects compared to administration of each molecule individually [37]. Tea, a potent antioxidant with metal chelating properties owing to its numerous hydroxyl groups $[38,39]$ has 
been shown to have neuroprotective effects in a number of neuropathological conditions linked to excitotoxicity and oxidative stress [40]. A few studies have reported significant beneficial effects of plant extracts including tea against metal-induced neurotoxicity [19, 41, 42, 43, 44]. However, to the best of our knowledge, this is the first study to compare the protective effects of polyphenol rich tea (Camellia sinensis) and EDTA against Cdinduced neurotoxicity.

The detrimental consequences of $\mathrm{Cd}$ exposure on the antioxidant defense system in the brain and its possible mechanisms as well as the protective effect of EDTA have been studied [45]. In the current study, the postulation that a natural product such as tea, offers more or comparable protective effects to that provided by the synthetic chelator $\mathrm{CaNa}_{2} \mathrm{EDTA}$ in reversing $\mathrm{CdCl}_{2}$ induced neurotoxicity was explored.

Results from our study indicate that the levels of reduced glutathione (GSH) and lipid peroxidation product malondialdehyde (MDA) in the brain were significantly increased following Cd exposure. The increase in GSH is a compensatory mechanism that is meant to counteract $\mathrm{Cd}$ induced oxidative stress [46, 47]. This is achieved by maintaining a high concentration of GSH in the cells through synthesis [48]. The finding that MDA was significantly increased in the brain following $\mathrm{Cd}$ challenge corroborates previous findings from other studies and is linked to the high amount of polyunsaturated fatty acids in the brain and its high oxygen turnover with concomitant $\mathrm{H}_{2} \mathrm{O}_{2}$ production [49].

In our study, the subcutaneous administration of $\mathrm{CdCl}_{2}$ to the rats caused a significant decrease in the levels of ZHX1 when compared to the control group. Cadmium can replace zinc in many biological systems [50] due to their similar oxidation states, and therefore the decrease observed in ZHX1 in the brain can be attributed to the substitution of $\mathrm{Zn}^{2+}$ for $\mathrm{Cd}^{2+}$ in the zinc finger motif with subsequent degradation of the mutant protein via the ubiquitin proteasome pathway [51]. Consequently, dysfunctions in the ZHX family members, and especially ZHX1 whose expression is slightly higher in the brain, results in the development and progression of neurodegenerative disorders observed in the current study as damage of the cerebral cortex manifested by a marked presence of lymphocytic inflammatory changes and brain necrosis.

In this study, $\mathrm{CdCl}_{2}$ enhanced GFAP that was characterized by round shaped cell bodies and relatively small number of fibrous processes implying astroglial activation and gliosis. This severe activation of astrocytes is associated with an ongoing neuroinflammatory response and neurodegenerative processes in the brain. This is in agreement with several other studies that observed that $\mathrm{CdCl}_{2}$ increased the expression of GFAP [52], with morphological alterations in GFAP-expressing astrocytes [53].

In line with our hypothesis, our findings demonstrated that oral administration of black tea extracts (BTE) and green tea extracts (GTE) significantly alleviated the symptoms of $\mathrm{CdCl}_{2}$ induced toxicity in the brain of rats. The most interesting finding in the present study was that the tea extracts significantly modulated the severe brain injuries manifested by significant increases in GSH, MDA and GFAP expression induced by $\mathrm{CdCl}_{2}$ as well as decreases in ZHX1 to levels that were comparable to those of the control rats. The tea extracts also reduced the marked presence of lymphocytic inflammatory changes and brain necrosis. These observations show that BTE and GTE have the ability of maintaining the endogenous antioxidants by scavenging the ROS-induced by cadmium. We hypothesize that the mechanisms of preventing and modulating cellular redox state due to $\mathrm{Cd}$ induced toxicity in the brain by the tea extracts may be through their ability to restore the activity of antioxidant enzymes, reduction of free 
radicals generation, termination of the initiation and propagation of lipid peroxidation, metal chelation and through activation of redox sensitive transcription factors and antioxidant enzymes $[54,55]$.

Consistent with our findings, previous studies have reported the neuroprotective effects of tea against neuronal damage in global ischemia in gerbils [56] by reducing lipid peroxidation in hydrocephalus-induced periventricular oxidative damage in murine model [57]. The ability of tea catechins (flavonoids) to act as antioxidants has been demonstrated to be associated with its metal chelating capacity and the potent capacity to quench singlet oxygen species [58], inhibition of peroxynitrite-mediated oxidation of dopamine and nitration of tyrosine residues [59]. Tea catechins which are water soluble antioxidants may chelate iron and copper, inhibit the generation of ROS and reduce the free form of iron and the mobility of the free radicals into the lipid. Furthermore, it has been shown that consumption of tea protects against hippocampal injury during transient global ischemia, and prevents nigral damage induced by xenobiotics [60, 61]. On the other hand, the protective effects of BTE in reducing $\mathrm{CdCl}_{2}$ induced toxicity could be attributed to the ability of theaflavins to reduce lipid accumulation [62] as well as in stabilizing the integrity of the cell membrane and keeping it intact [63].

It is worth noting that for tea flavonoids to modulate the effects of xenobiotics in the brain, they must have the ability of crossing the blood brain barrier (BBB) which controls entry of such molecules into the brain [64]. Results from this study suggest that the protective effects of the orally administered tea extracts could be due to their ability of crossing the blood brain barrier. This is consistent with previous studies that have demonstrated that tea polyphenols are found in the brain after their oral administration $[65,30]$. This capability of tea polyphenols to cross the $\mathrm{BBB}$ and their localization in the brain makes them better candidates for direct neuroprotective and neuromodulatory actions.

A very outstanding observation in the current study was that the protective effects of both the BTE and GTE from cultivar TRFK 6/8 against the Cd induced neurotoxicity were not significantly different. These may be explained by the fact that flavonols are less affected by tea processing and are present in almost comparable amounts in both green and black teas processed from polyphenol rich cultivars [66]. Theaflavins present in black tea possess almost the same antioxidant potency as catechins present in green tea, and the conversion of catechins to theaflavins during the auto-oxidation step (fermentation) of black tea processing does not significantly alter their free radical-scavenging activity [67].

Our results demonstrate clearly that both black and green tea extracts out competed EDTA which is a synthetic chelating antioxidant of great repute in protecting the brain against $\mathrm{CdCl}_{2}$ induced injury. This observation may be attributed to the inefficiency of EDTA as an antioxidant as well as its relatively poor chelating properties due to its structure that gives incomplete shielding of $\mathrm{Fe}^{3+}$, forming an open complex (basket complex) that increases the catalytic capacity of $\mathrm{Fe}^{3+}$ for generating oxidative stress [68]. Additionally, EDTA is distributed mainly in the extracellular fluids, which limits its capacity to chelate out metals from inside the cells with consequences of redistributing heavy metals from other tissues to the brain [16].

Though we established significant trends indicative of the efficacy of tea extracts as metal chelators using five rats per group, we did not study the effect of rat sample size on these trends and we propose that this should be determined in order to estimate the required sample size for future studies. 


\section{Conclusion}

This paper reports for the first time that tea extracts are better than EDTA in protecting rats against cadmium induced toxicity and disturbances of antioxidant defense system in the brain. The efficacy of tea surpasses that of EDTA, the conventional chelating agent. Tea extracts may therefore reduce neurodegeneration induced by cadmium and promote brain health in humans.

Abbreviations: $\mathrm{CdCl}_{2}$-Cadmium chloride, BBB- blood brain barrier, $\mathrm{CaNa}_{2} \mathrm{EDTA}$ - Calcium disodium ethylenediamine tetra acetic acid, BAL- British Anti Lewisite, DMPS- Sodium 2, 3dimercaptopropane 1-sulfonate, DMSA- Meso 2, 3-dimercaptosuccinic acid, ZHX1- Zinc Fingers and Homeoboxes Protein 1, TBARS- Thiobarbituric Acid Reactive Substances, GSHreduced glutathione, CNS- Central nervous system, NADPH- Reduced nicotinamide adenine dinucleotide phosphate, DNMT3B - DNA methyltransferase B , SOP- Standard Operating Procedures, MDA- Malondialdehyde, GT- Green tea, BT- Black tea, ROS- Reactive oxygen species.

\section{Acknowledgements}

We thank all those colleagues who contributed to this study. We thank Egerton University, Tea Research Institute (TRI) and Primate Research Institute (PRI) for providing facilities to conduct the research.

\section{Disclosure statements}

Availability of data and materials: Data are contained within the paper.

Authors' contributions: WFN, AGO, KR, NRM and KSM conceived and designed the study. WFN, NRM, WJK and NM supervised AGO, KR, MF, NN and MKO carry out experiments and collect data. AGO and KR validated the data collected and analyzed it statistically. AGO prepared the first draft of the manuscript which was reviewed and revised by WFN, KR, NRM, and KSM. Improved drafts of the manuscript were reviewed by all the authors. All authors have read and approved the final version of the manuscript.

Competing interests: The authors declare that they have no competing or conflicting interests.

Ethics approval: All rat studies were performed using protocols approved by the Institutional Animal Care and Use Committee (IACUC) of the Institute of Primate Research (IPR), with approval number IRC/08/13.

Funding: This study was funded by the National Council for Science and Technology (NCST) of Kenya through Grant Number NCST/ST\&I/003/3 ${ }^{\text {rd }}$ STI CALL/51.

\section{References}

1. Nordberg GF, Nogawa K, Nordberg M, Friberg LT: Foreword: Metals - A new old environmental problem and Chapter 23: Cadmium. In Handbook on the Toxicology of Metals, 3rd ed.; Nordberg, G.F., Fowler, B.A., Nordberg, M. and Friberg, L.T., Eds.; Academic Press: Burlington, MA, USA, 2011; pp. vii, 446-451, 463-470, 600-609. 
2. Flora SJS, Mittal M, Mehta A: Heavy metal induced oxidative stress and its possible reversal by chelation therapy. Indian Journal of Medical Research, 2008, 128: 501-523.

3. Rai A, Maurya SK, Khare P, Shrivastava A, Bandyopadhyay S: Characterization of developmental neurotoxicity of $\mathrm{As}, \mathrm{Cd}$ and $\mathrm{Pb}$ mixture: Synergistic action of metal mixture in glial and neuronal functions. Toxicological Science, 2010, 118:596-601.

4. Amara, S, Douki, T, Garrel, C, Favier, A, Ben Rhouma, K, Sakly, M, Abdelmelek, H: Effects of static magnetic field and cadmium on oxidative stress and DNA damage in rat cortex brain and hippocampus. Toxicology and Industrial Health, 2011, 27: 99-106.

5. Tobwala S, Wang H, Carey WJ, Banks AW, Ercal, N: Effects of Lead and Cadmium on brain endothelial cell survival, monolayer permeability and crucial oxidative stress markers in an in vitro model of the Blood-Brain Barrier. Toxics, 2014, 2: 258-275.

6. Waalkes MP, Coogan TP, Barter RA: Toxicological principles of metal carcinogenesis with special emphasis on cadmium. Critical Review in Toxicology, 1992, 22:175-201.

7. Chen L, Xu B, Liu L, Zhou HLY, Chen W, Han XST, Kontos DC, et al.: Cadmium induction of reactive oxygen species activates the mTOR pathway, leading to neuronal cell death, Free Radical Biology and Medicine, 2011, 50: 624-632.

8. Sarkar S, Yadav P, Bhatnagar D: Cadmium-induced lipid peroxidation and the antioxidant system in rat erythrocytes: The role of antioxidants. Journal of Trace Elements in Medicine and Biology, 1997, 11: 8-13.

9. Kim S, Park J, Choi M, Kim H, Park H, Jung Y, Lee J, et al.: Zinc-fingers and homeoboxes 1 (ZHX1) binds DNA methyltransferase (DNMT) 3B to enhance DNMT3Bmediated transcriptional repression, Biochemical and Biophysical Research Communications, 2007, 355:318-323.

10. Hartwig A: Zinc finger proteins as potential targets for toxic metal ions: differential effects on structure and function. Antioxidants and Redox Signaling, 2001, 3: 625-634.

11. Hanas JS and Gunn CG. Inhibition of transcription factor IIIA-DNA interactions by xenobiotic metal ions. Nucleic Acids Research, 1996, 24: 924-930.

12. Chen L, Liu L, Huang S: Cadmium activates the mitogen activated protein kinase (MAPK) pathway via induction of reactive oxygen species and inhibition of protein phosphatases 2A and 5, Free Radical Biology and Medicine, 2008, 45:1035-1044.

13. Antonio M, Corpas I, Laret ML: Neurochemical changes in newborn rats brain after gestational cadmium and lead exposure. Toxicology Letters, 1999, 104: 1-9.

14. Brahmachari S, Yiu K, Fung KY, Pahan K: Induction of Glial Fibrillary Acidic Protein Expression in Astrocytes by Nitric Oxide Journal of Neuroscience, 2006, 3:4930-4939.

15. Quinlan RA, Brenner M, Goldman JE, Messing A: GFAP and its role in Alexander disease. Experimental Cell Research, 2007, 313: 2077-2087.

16. Flora SJS, Pachauri V: Chelation in metal intoxication. International Journal of Environmental Research and Public Health, 2010, 7: 2745-2788.

17. Yan H, Carter CE, Xu C, Singh PK, Jones MM, Johnson JE, Dietrich MS: Cadmiuminduced apoptosis in the urogenital organs of the male rat and its suppression by chelation. Journal of Toxicology and Environmental Health, 1997, 52:149-168

18. Lin Tan DT, Lin JL, Yen TH. Chen KH, Huang YL: Long-term outcome of repeated lead chelation therapy in progressive non-diabetic chronic kidney diseases. Nephrology Dialysis and Transplant, 2007, 22: 2924-2931. 
19. Mandel S, Youndin, MB: Catechin polyphenols: neurodegeneration and neuroprotection in neurodegenerative diseases. Free Radical Biology and Medicine, 2004, 37:304-317.

20. Mandel SA, Avramovich-Tirosh Y, Reznichenko L, Zheng H, Weinreb O, Amit T, Youdim MB: Multifunctional activities of green tea catechins in neuroprotection. Neurosignals, 2005, 14: 46-60.

21. Karori SM, Ngure RM, Wachira FN, Wanyoko JK, Mwangi JN: Different types of tea products attenuate inflammation induced in Trypanosoma brucei infected mice, Parasitology International, 2008, 57:325-33.

22. Rietveld AWS: Antioxidant effects of Tea: Evidence from human clinical trials Journal of Nutrition, 2003, 133: 3285S-3292S,

23. Kumamoto M, Sonda T, Nagayama K, Tabata M: Effects of $\mathrm{pH}$ and metal ions on antioxidative activities of catechins. Bioscience Biotechnology and Biochemistry, 2001, 65:126-132.

24. Jung JY, Han CR, Jong J, Kim HJ, Lim HS, Lee HS, Park HO, et al.: Epigallocatechin gallate inhibits nitric oxide induced apoptosis in rat PC12 cells. Neuroscience Letters, 2007, 41: 22-227.

25. Farhoosh R, Golmovahhed GA, Khodaparast MH: Antioxidant activity of various extracts of old tea leaves and black tea wastes (Camellia sinenses L.). Food Chemistry, 2007, 100: 231-236.

26. Rajeswari A, Sabesan M: Inhibition of monoamine oxidase-B by the polyphenolic compound, curcumin and its metabolite tetrahydrocurcumin, in a model of Parkinson's disease induced by MPTP neurodegeneration in mice. Inflammopharmacology, 2008, 16:96-99.

27. Wang Q, Sun AY, Simonyi A, Miller DK, Smith RE, Luchtefeld RG, Korthuis RJ, et al.: Oral administration of grape polyphenol extract ameliorates cerebral ischemia/reperfusion-induced neuronal damage and behavioral deficits in gerbils: Comparison of pre- and post-ischemic administration. Journal of Nutritional Biochemistry, 2009, 20: 369-377.

28. Koech KR, Wachira FN, Karori SM: In vitro antimicrobial and synergistic properties of water soluble green and black tea extracts, African Journal of Microbiology Research, 2014, 8:1527-1534.

29. Rahman I, Kode A, Biswas SK: Assay for quantitative determination of glutathione and glutathione disulfide levels using enzymatic recycling method, Nature protocols, 2006, 1:3159-3165

30. Rashid K, Wachira FN, Nyabuga JN, Wanyonyi B, Murilla G, Isaac AO: Kenyan purple tea anthocyanins ability to cross the blood brain barrier and reinforce brain antioxidant capacity in mice. Nutritional Neuroscience, 2014, 17:178-185.

31. Zhu Y, Romero MI, Ghosh P, Ye Z, Charnay P, Rushing EJ, Marth JD, et al.: Ablation of NF1 function in neurons induces abnormal development of cerebral cortex and reactive gliosis in the brain. Genes and Development, 2001, 15: 859-876.

32. Sinha RA, Khare P, Rai A, Maurya SK, Pathak A, Mohan V, Nagar GK, et al.: Antiapoptotic role of omega-3-fatty acids in developing brain: Perinatal hypothyroid rat cerebellum as apoptotic model. International Journal of Developmental Neuroscience, 2009, 27: 377-383. 
33. Otani A, Takagi H, Oh H, Koyoma S, Matsumura M, Honda Y: Expressions of angiopoietins and Tie2 in human choroidal neovascular membranes Investigative Opthalmology and Visual Science, 1999, 40:1912-1920.

34. Wright RO and Baccarelli A: Metals and neurotoxicology. Journal of Nutrition, 2007, 137: 2809-2813.

35. Saljooghi, AS and Fatemi, SJ: Clinical evaluation of Deferasirox for removal of cadmium ions in rat Biometals, 2010, 23:707-712.

36. Jamilaldin FS, Amir SS, Faezeh DB, Iranmanesh M, Mohammad RG: Chelation of cadmium by combining deferasirox and deferiprone in rats. Toxicology and Industrial Health, 2011, 27: 371-377.

37. Gil HW, Kang EJ, Lee KH, Yang JO, Lee EY, Hong SY: Effect of glutathione on the cadmium chelation of EDTA in a patient with cadmium intoxication. Human and Experimental Toxicology, 2011, 30:79-83.

38. Umeno A, Horie M, Murotomi K, Nakajima Y, Yoshida Y: Antioxidative and antidiabetic effects of natural polyphenols and isoflavones, Molecules, 2016, 21:1-15.

39. Fatima M, Kesharwani KR, Misra K, Rizvi SI: Protective Effect of Theaflavin on Erythrocytes Subjected to In Vitro Oxidative Stress, Biochemistry Research International, 2013, 2013:1-7.

40. Weinreb O, Amit T, Mandel S, Youdim MB: Neuroprotective molecular mechanisms of (-)-epigallocatechin-3-gallate: A reflective outcome of its antioxidant, iron chelating and neuritogenic properties. Genes Nutrition, 2009, 4: 283-296.

41. Sethi, P, Jyoti, A, Hussain, E, Sharma, D: Curcumin attenuates aluminium induced functional neurotoxicity in rats. Pharmacology Biochemistry and Behavior, 2009, 93:3139.

42. Abib RT, Peres KC, Barbosa AM, Peres TV, Bernardes A, Zimmermann LM, Quincozes SA, et al.: Epigallocatechin-3-gallate protects rat brain mitochondria against cadmiuminduced damage. Food and Chemical Toxicology, 2011, 49: 2618-2623.

43. Abib RT, Quincozes-Santos, A, Nardin P, Wofchuk, ST, Perry, ML, Goncalves CA, Gottfried C: Genoprotective effects of the green tea-derived polyphenol/epicatechin gallate in C6 astroglial cells. Journal of Medicinal Food, 2010, 13:1111-1115.

44. Maodaa SN, Allam AA, Ajarem J, Abdel-Maksoud MA, Al-Basher GI, Wang ZY: Effect of parsley (Petroselinum crispum, Apiaceae) juice against cadmium neurotoxicity in albino mice (Mus Musculus). Behavioral and Brain Functions, 2016, 12, 6, doi:10.1186/s12993-016-0090-3.

45. Mikirova N, Casciari J, Hunninghake R: Efficacy of oral DMSA and intravenous EDTA in chelation of toxic metals and improvement of the number of stem/ progenitor cells in circulation. iMedPub Journals, 2011, 2:1-8.

46. Antonio MT, Corredor L, Leret ML: Study of the activity of several brain enzymes like markers of the neurotoxicity induced by perinatal exposure to lead and/or cadmium. Toxicology Letters, 2003, 143: 331-340.

47. Sato Y, Itagaki S, Kurokawa T: In vitro and in vivo antioxidant properties of chlorogenic acid and caffeic acid International Journal of Pharmaceutics, 201, 403: 136-138.

48. Martin HL, Teismann P: Glutathione-review on its role and significance in Parkinson's disease, The FASEB Journal, 2008, 23:3263-3272. 
49. Bultel-Poncé V, Durand T, Guy A, Oger C, Galano JM: Non enzymatic metabolites of polyunsaturated fatty acids:friend or foe, Oilseeds \& fats Crops and Lipids, 2016, 23:D118(1-10).

50. Jaishankar M, Tseten T, Anbalagan N, Mathew BB, Krishnamurthy N, Beeregowda NK: Toxicity, mechanism and health effects of some heavy metals, Interdisciplinary Toxicology, 2014, 7:60-72.

51. Chen S, Yu X, Lei Q, Ma L, Guo D: The SUMOylation of zinc-fingers and homeoboxes 1 (ZHX1) by Ubc9 regulates its stability and transcriptional repression activity, Journal of Ccellular Biochemistry, 2013, 114:2323-2333.

52. Kovalchuk YP, Prischepa IV, Si U, Nedzvetsky VS, Kot YG, Persky EE, Ushakova VA: Distribution of glial fibrillary protein in different parts of the rat brain under cadmium exposure, The Ukranian Biochemical Journal, 2015, 87:116.123.

53. McCall MA, Gregg RG, Behringer RR, Brenner M, Delaney CL, Galbreath EJ, Zhang CL, et al.: Targeted deletion in astrocyte intermediate filament (GFAP) alters neuronal physiology. Proceedings of the National Academy of Sciences of the United States of America, 1996, 93: 6361-6366.

54. Higdon JV, Frei B: Tea catechins and polyphenols: Health effects, metabolism, and antioxidant functions. Critical Reviews in Food Science and Nutrition, 2003, 43: 89-143.

55. Mohamadin AM, El-Beshbishy HA, El-Mahdy MA: Green tea extracts attenuate cyclosporine A-induced oxidative stress in rats Pharmacological Research, 2005, 51:5157.

56. Lee SR, Lim KJ, Suh SI and Jung JG: Protective effect of green tea polyphenol (-) epigallocatechin gallate and other antioxidants in gerbil brain homogenates. Phytotherapy Research, 2003, 17:206-209.

57. Etus V, Altug AB, Ceylan S: Green tea polyphenol (-) epigallocatechin gallate prevents oxidative damage on perivascular white matter of infantile rats. Tohoku Journal of Experimental Medicine, 2003, 200: 203-209.

58. Tournaire C, Croux S, Maurette MT, Beck I, Hocquaux M, Braun AM, Oliveros E: Antioxidant activity of flavonoids: Efficiency of singlet oxygen (1 delta g) quenching. Journal of Photochemistry and Photobiology, 1993, B 19: 205-215.

59. Kerry N, Rice-Evans C: Inhibition of peroxynitrite-mediated oxidation of dopamine by flavonoid and phenolic antioxidants and their structural relationships. Journal of Neurochemistry, 1999, 73: 247-253.

60. Levites Y, Weinreb O, Maor G: Green tea polyphenol ( \pm )-epigallocatechin-3-gallate prevents N-methyl-4-phenyl-1,2,3,6 tetehydropyridine induced dopaminergic neurodegeneration Journal of Neurochemistry, 2001, 78:1073-1082.

61. Levites Y, Youdim MB, Maor G: Attenuation of 6-hydroxydopamine (6-OHDA)-induced nuclear factor kappa B (NF-kB) activation and cell death by tea extracts in neuronal cultures. Biochemical Pharmacology, 2002, 63: 21-29.

62. Tandon SK, Singh S, Prasad S, Srivastava S, Siddiqui MK: Reversal of lead-induced oxidative stress by chelating agent, antioxidant, or their combination in the rat. Environmental Research, 2002, 90: 61-66.

63. Waisberg M, Joseph P, Hale B, Beyersmann, D: Molecular and cellular mechanisms of cadmium carcinogenesis. Toxicology, 2003, 192: 95-117. 
64. Abbott NJ: Astrocyte endothelial interactions and brain barrier permeability. Journal of Anatomy, 2002, 200: 629-638

65. Abd El, Mohsen MM, Kuhnle G, Rechner AR: Uptake and metabolism of epicatechin and its access to the brain after oral ingestion. Free Radical Biology and Medicine, 2002, 33: 1693-1702.

66. Hossain MA, Russell JC, Miknyoczki S, Ruggeri B, Lal B, Laterra J: Vascular endothelial growth factor mediates vasogenic edema in acute lead encephalopathy. Annals of Neurology, 2004, .55: 660-667.

67. Shukla A, Shukla GS, Srimal RC: Cadmium-induced alterations in blood-brain barrier permeability and its possible correlation with decreased microvessel antioxidant potential in rat. Human Experimental Toxicology, 1996, 15: 400-405.

68. Singh, S, Khodr, H, Tayler, MI, Hider, RC: Therapeutic iron chelators and their potential side-effects. Biochemical Society Symposium, 1995, 61: 127-137. 